\title{
Traumatic Experiences Disrupt Amygdala - Prefrontal Connectivity
}

\author{
Dong Hoon Oh
}

Additional information is available at the end of the chapter

http://dx.doi.org/10.5772/48691

\section{Introduction}

Psychological "trauma" refers to a sudden intense surge of anxiety secondary to some external event that exceeds the subject's ability to cope with and to defend against. However, the term "trauma" is actually difficult to define due to its subjective nature. The American Psychiatric Association defines it as "an event or events that involves actual or threatened death or serious injury, or a threat to the physical integrity of self or others." Examples of such events include military combat, violent personal attack, natural or humanmade disasters, and torture. For children, sexually traumatic events may include ageinappropriate sexual experiences without violence or injury (American Psychiatric Association. and American Psychiatric Association. Task Force on DSM-IV, 1994).

Unfortunately, traumatic events happen to people all over the world. Estimates of the prevalence of traumatic experiences are likely to vary with the method of assessment (Breslau, 2002, Breslau et al., 1998, Copeland et al., 2007, Finkelhor et al., 2005, Frans et al., 2005, Helzer et al., 1987). In particular, recent epidemiological studies have demonstrated high rates $(70 \%-80 \%)$ of lifetime traumatic experiences, suggesting that previous epidemiological surveys may have underestimated the prevalence of traumatic events (de Vries and Olff, 2009, Mills et al., 2011). Although all subjects exposed to traumatic events do not come to exhibit mental disorders, childhood trauma increases the risk of mental disorders during adulthood (Gilbert et al., 2009). Therefore, it is clinically important to understand the biological and psychological changes induced by trauma.

Traumatic experiences induce a wide range of physical and psychological symptoms that affect all aspects of life for survivors. These symptoms can involve clinically significant distress and impairment in social, occupational, and other areas of functioning. Traumatic experiences also lead to significant structural and functional changes in brain regions implicated in emotional and cognitive processing. These brain areas include the medial 
prefrontal cortex (mPFC), which contains the anterior cingulate cortex (ACC) (Ansell et al., 2012), the hippocampus, and the amygdala (Bremner, 2006, Kolassa et al., 2007, Shin et al., 2006).

Recent advances in neuroimaging have revealed anatomical and functional connectivity between the amygdala and the prefrontal cortex, which is dedicated to emotion regulation. There is accumulating evidence that the amygdala and the prefrontal cortex play critical roles in conditioning and the extinction of memories of traumatic fear. The prefrontal cortex regulates stress-induced fear and anxiety-like behaviors via inhibitory effects on amygdala output and processing (Akirav and Maroun, 2007, Bishop, 2007).

In this chapter, we will describe the structural (volumetric) and functional changes in the amygdala and prefrontal cortex resulting from traumatic stresses. We will argue that there is a structural and functional disconnection between the amygdala and the prefrontal cortex in patients with trauma-related psychiatric disorders but not in controls. We will then present evidence that traumatic experiences can disrupt the normal connectivity between the amygdala and the prefrontal cortex, which suggests that effective interactions between these two brain areas are needed for healthy outcomes of traumatic experiences.

\section{Trauma-related structural and functional changes in the amygdala}

Anatomically, the amygdala is an almond-shaped mass located above and in front of the temporal horn of the lateral ventricle and anterior to the tail of the caudate nucleus. It is a complex structure containing more than a dozen nuclei that are richly interconnected (Pessoa, 2010). The amygdala also has extensive connections with cortical and subcortical regions (Sah et al., 2003). Functionally, it is an essential component of the circuit involved in implicit emotional learning and memory, emotional modulation of memory, emotional influences on attention and perception, emotion and social behavior, and emotion inhibition and regulation (Phelps and LeDoux, 2005). In particular, the amygdala mediates the acquisition and expression of conditioned fear and the enhancement of emotional memory (Koenigs and Grafman, 2009).

\subsection{Traumatic experiences induce changes in amygdala volume}

A number of studies have provided evidence of effects of trauma on amygdala volume. Interestingly, the effects of traumatic stress on amygdala volume differ between children and adults. Childhood trauma is associated with increases in amygdala volume, whereas traumatic stress in adulthood is associated with reductions in amygdala volume. Two studies have detected larger amygdala volumes in children and adolescents who have experienced early institutional deprivation and subsequent adoption (Mehta et al., 2009, Tottenham et al., 2010). Mehta et al. (2009) reported larger amygdala volumes in 14 adoptee adolescents who had experienced severe early institutional deprivation in Romania than in a group of non-institutionalized controls $(n=11)$. The conditions of care in the Romanian institutions that these children had experienced varied from poor to appalling. Tottenham et 
al. (2010) demonstrated that children who had been adopted out of an orphanage at older ages (>15 months old) had larger amygdala volumes than early-adopted children $(<15$ months old) and non-adopted controls. Similarly, a recent study demonstrated that children exposed to maternal depression $(n=17)$ since birth had significantly larger amygdala volumes than controls $(n=21)$, whereas the two groups did not differ with respect to hippocampal volume (Lupien et al., 2011).

In contrast, the total volume of the amygdala in adult breast cancer survivors $(n=35)$ with a history of cancer-related intrusive recollections was significantly smaller than in control breast cancer survivors who had no such history $(n=41)$ (Matsuoka et al., 2003). A study using a voxel-based morphometry (VBM) method detected smaller amygdala volumes in healthy adults $(n=17)$ who were within a mile and a half of the World Trade Center on September 11, 2001, than in the comparison group ( $n=19)$ (Ganzel et al., 2008). Mollica et al. (2009) reported that South Vietnamese ex-political detainees exposed to torture and traumatic head injury had a higher rate of depression than those without traumatic head injury. Trauma/torture events were associated with bilateral loss of amygdala volume (Mollica et al., 2009).

The results of most recent meta-analyses indicate that trauma-related psychiatric disorders such as post-traumatic stress disorder (PTSD), major depressive disorder (MDD) and borderline personality disorder (BPD), are associated with reductions in amygdala volume in the adult. Sacher et al. (2012) analyzed 10 selected studies of adult patients with MDD and found a significant decrease in left amygdala volume in the MDD group. Woon and Hedges (2009) analyzed published data from nine studies comparing amygdala volumes in adult subjects with PTSD, and found no significant effect on amygdala volumes. However, another meta-analysis found significantly smaller left amygdala volumes in adults with PTSD than in either healthy or trauma-exposed controls (Karl et al., 2006). In addition, Nunes et al. (2009) in a meta-analysis of six studies demonstrated significantly reduced volumes of both right and left hippocampi and amygdalae in patients with BPD. One meta-analysis reported that amygdala volume in children with maltreatment-related PTSD did not differ from that in healthy controls (Woon and Hedges, 2008). Thus human data on amygdala volumes in patients with trauma-related psychiatric disorders have thus far yielded conflicting or varied results. Therefore, disease states (or courses), symptom severity and histories of antidepressant medication should be taken into consideration when interpreting the results of volumetric studies on the amygdala in patients with trauma-related psychiatric disorders.

\subsection{Traumatic experiences induce changes in amygdala function}

In human neuroimaging studies, amygdalar hyper-responsiveness to emotionally negative stimuli has been shown to be associated with trait anxiety (Etkin et al., 2004, Sehlmeyer et al., 2011), PTSD (Shin et al., 2005, Rauch et al., 2000, Francati et al., 2007) and MDD (Sheline et al., 2001, Siegle et al., 2007, Suslow et al., 2010).

A recent functional magnetic resonance imaging (fMRI) study of a large sample of healthy adults $(\mathrm{n}=148)$ showed that childhood maltreatment (Childhood Trauma Questionnaire 
scores) was positively associated with right amygdala responsiveness to negative facial expressions (Dannlowski et al., 2012). In addition, adverse early rearing environments in the postnatal period were followed by heightened amygdala activity during childhood. Tottenham et al. (2011) observed the brain activity of two groups of children while performing an Emotional Face Go/No-Go task. The scanned fMRI obtained in the previously institutionalized group of children showed enhanced activity in the amygdala in comparison to the control (Tottenham et al., 2011). Similarly, the results of another fMRI study comparing unipolar depressed patients with and without a history of significant early life trauma $(n=20)$ and healthy subjects $(n=16)$ provided a robust positive correlation between physical abuse and right amygdalar responses (Grant et al., 2011). Since childhood trauma is a predisposing factor for PTSD and adult depression, the results of these studies suggest that heightened amygdalar response is a mediator between childhood trauma and the development of trauma-related psychiatric disorders such as PTSD and MDD.

\section{Trauma-related structural and functional changes in the prefrontal cortex}

The prefrontal cortex consists of three major anatomical regions: the dorsolateral prefrontal cortex (dlPFC), the orbitofrontal cortex (OFC), and the medial prefrontal cortex (mPFC). The dorsolateral prefrontal cortex is a multimodal association area that participates in higher cognitive functions (e.g., executive functions), whereas the OFC and the $\mathrm{MPFC}$ are considered to make up the limbic (or paralimbic) area that participates in emotional and motivational functions (Ichihara-Takeda and Funahashi, 2007). In particular, the ventromedial prefrontal cortex (vmPFC) mediates the extinction of conditioned fear and the volitional regulation of negative emotion (Koenigs and Grafman, 2009).

\subsection{Traumatic experiences induce structural changes in the prefrontal cortex}

There are mixed results from studies comparing the volumes of the prefrontal cortex in children with maltreatment-related PTSD and those in non-maltreated children (McCrory et al., 2011). However, reduced prefrontal volume in adults with traumatic experiences has been a consistent finding. For examples, a previous VBM study that compared the gray matter volume of cancer survivors with PTSD with the gray matter volume of those without PTSD and of healthy subjects demonstrated that the gray matter volume of the right OFC was significantly smaller in cancer survivors with PTSD $(n=9)$ than in those without PTSD $(n=67)$ or healthy subjects ( $n=70)$ (Hakamata et al., 2007). Chronic exposure to harsh corporal punishment was associated with a marked reduction in gray matter volume in the right $\mathrm{mPFC}$ in young adults (18-25 years). There were also possible associations between harsh corporal punishment and reduced gray matter volume in the left dIPFC and the right ACC (Tomoda et al., 2009). Another recent VBM study examined whether healthy control subjects and unmedicated patients with depression and/or anxiety disorders who reported childhood emotional maltreatment before age $16(\mathrm{n}=84)$ displayed structural brain changes compared with control subjects and patients who reported no childhood abuse $(n=97)$. This 
study showed that self-reported childhood emotional maltreatment is associated with a profound reduction of $\mathrm{mPFC}$ volume, even in the absence of physical or sexual abuse during childhood (van Harmelen et al., 2010). A longitudinal multiwave neuroimaging study in a cohort of direct survivors of a South Korean subway disaster (2003) was conducted as a five-year follow-up case-control study. This study demonstrated that disaster survivors early in the course of PTSD had greater cortical thickness in the dIPFC regions than controls. This greater dlPFC thickness early after the trauma, which was associated with earlier improvement and subsequent recovery from PTSD, gradually reverted to the level of controls (Lyoo et al., 2011).

Based on the results of most recent meta-analyses, prefrontal cortical volume reduction is frequently involved in both MDD and PTSD. A meta-analysis study of 41 studies found significant volume reductions in the prefrontal cortex (especially the OFC) and ACC of patients with MDD (Ansell et al. 2012). In particular, the subgenual ACC and OFC were significantly smaller in antidepressant-free patients compared with medicated patients (Bora et al., 2012). A previous meta-analysis also found significantly smaller ACC in adults with PTSD than in trauma-exposed controls (Karl et al., 2006).

Changes in gray matter volume are associated not only with trauma-related psychiatric disorders but also with recent adverse life events and perceived stress; these associations suggest that some trauma-related changes in gray matter volume may act as vulnerability markers that precede the presence of trauma-related psychiatric disorders (Ansell et al., 2012). However, the exact mechanism that causes prefrontal volume reduction in patients with trauma-related psychiatric disorders and after traumatic events remains unknown. Acute or chronic traumatic stress may induce gray matter volume reduction by several possible mechanisms including glucocorticoid-induced neuronal cell damage, glutamatemediated neuronal cell death (apoptosis) and decreased neurogenesis (Zhu et al., 2006, Oh et al., 2012).

\subsection{Traumatic experiences induce functional changes in the prefrontal cortex}

It is known that trauma-related psychiatric disorders are associated with functional abnormalities in the prefrontal cortex. The prefrontal cortex is involved in various affective and cognitive functions supporting the processing of traumatic memories. Traumatic experiences induce structural and functional abnormalities in the prefrontal area, and these are implicated in deficient traumatic memory processing and the subsequent development of trauma-related symptoms (McFarlane et al., 2002).

A recent study compared twelve adopted adolescents who suffered from deprivation of early caregivers (early-life stress group, 9 females) with 21 healthy control adolescents (10 females) who lived with their biological parents. The subjects were tested using a cognitive control task and analyzed using fMRI. The early-life stress group took longer to switch from a prepotent response to an alternative response than the control group. Observation of neural activity revealed greater activation of several regions involved in cognitive control including the dIPFC and the striatum in the early-life stress group than in controls (Mueller 
et al., 2010). A previous study investigating the functional neuroanatomical correlates of response inhibition in thirty right-handed medication-naive youths (10-16 years, $n=16)$ with post-traumatic symptoms (PTSS) and in a gender-matched control group of healthy youths $(n=14)$ found that the PTSS subjects performed similarly to the control subjects in the Go/NoGo task. However, during the Go-No/Go task, the PTSS group had greater medial frontal activation than the controls (Carrion et al., 2008).

A recent fMRI study in adults examined the relationship between recent negative life stress and regional brain activity in adult subjects with MDD $(n=15)$ and in individually matched healthy controls $(n=15)$. No significant effects of stress on brain activation in response to negative words were found in the controls. However, in the MDD group, negative correlations were found in the right ventrolateral prefrontal cortex (vlPFC), the subgenual cingulate area and the nucleus accumbens. Positive correlations were also found bilaterally in the orbitofrontal areas (Hsu et al. 2010). A previous fMRI study investigated the neural activation patterns during recall of autobiographical traumatic episodes in BPD patients compared with recall of negative (aversive) but nontraumatic episodes. Contrasting between trauma and nontrauma conditions revealed activation of the OFC and Broca's area in all the subjects, as well as activation of the occipital-mesial and temporal-anterior areas (Driessen et al., 2004).

In summary: structural neuroimaging studies have identified volumetric changes in the amygdala and the prefrontal cortex in some subjects exposed to traumatic events. Functional neuroimaging has also revealed activation abnormalities in the same areas in traumatized subjects. These findings have prompted neuroimaging studies aimed at discovering the relationship and interactions between the amygdala and the prefrontal cortex.

\section{Trauma and amygdala-prefrontal connectivity}

Using the diffusion tensor imaging (DTI) method, it is possible to delineate non-invasively the structural connections between the amygdala and the prefrontal cortex in humans. The amygdala is extensively interconnected with the prefrontal cortex, especially with the OFC (Croxson et al., 2005). Numerous studies have shown that trauma-related psychiatric disorders are associated with abnormal interactions between the amygdala and the prefrontal area (Etkin and Wager, 2007). The prefrontal cortex is thought to be involved in top-down regulation of the amygdala, while the amygdala in turn modulates prefrontal cortical activity. Clarifying the exact neurobiological mechanism of the amygdala-prefrontal dynamic interaction is essential for understanding the pathophysiology of trauma-related psychiatric disorders and for treatment of these disorders.

\subsection{Traumatic experiences disrupt amygdala-prefrontal connectivity}

The amygdala and the prefrontal cortex are not only structurally but also functionally interconnected. Traumatic experiences induce structural and functional changes in the amygdala and prefrontal cortex, respectively. Changes in amygdala-prefrontal interactions 
may induce deficits in emotional processing. For examples, a recent DTI study showed that individual differences in trait anxiety scores were negatively correlated with the mean fractional anisotropy (FA) value of the entirety of the identified amygdala-vmPFC pathway. This result suggests that the strength of the anatomical amygdala-prefrontal pathway predicts lower levels of normal trait anxiety (Kim and Whalen, 2009). A previous study analyzed structural and functional MRI data collected from a sample of 20 healthy subjects. It found that greater vmPFC gray matter thickness was associated with greater reductions in activation of the left amygdala during an affect-labeling task. This supports the idea that the vmPFC has a general role in suppression of amygdala activity (Foland-Ross et al., 2010).

Changes in amygdala-prefrontal interactions are involved in the pathophysiology of trauma-related psychiatric disorders. In a positron emission tomography (PET) study examining glucose metabolism, there were significant positive correlations between right OFC and ventral amygdala in healthy control subjects $(n=24)$. BPD patients $(n=26)$ had weak correlations between amygdala and the anterior PFC. This study demonstrated a tight coupling of metabolic activity between right OFC and ventral amygdala in healthy control subjects that was not present in BPD patients (New et al., 2007). Patients with MDD were characterized by decreased interaction between the amygdala and the prefrontal areas (dorsal ACC and dIPFC) during emotional processing compared with healthy subjects. Amygdala-prefrontal connectivity is significantly correlated with the severity of depression. These results suggest that patients with reduced amygdala-dlPFC interaction have a longer and more severe course of disease (Dannlowski et al., 2009). A recent fMRI study showed that both medication-free MDD patients $(n=29)$ and never-depressed subjects with cognitive vulnerability to depression $(n=26)$ displayed similar responses to emotional stimuli, with significantly lower activity in the dIPFC and significantly greater activity in the amygdala than healthy control subjects ( $\mathrm{n}=31$ ) (Zhong et al., 2011).

Numerous neuroimaging studies of PTSD have also revealed a pattern of hyperactivation in regions involved in the generation of emotion (the amygdala and the insula) and corresponding hypoactivation in regions involved in the regulation of emotion (the mPFC and the ACC). Actually, relative to non-traumatized controls, subjects with PTSD showed a marked reduction in activity in the $\mathrm{MPFC}$ and a small but significant enhancement in left amygdala activity in response to overtly presented fearful face stimuli. This suggests that traumatic life events disrupt the normal pattern of $\mathrm{mPFC}$ and amygdala regulation (Williams et al., 2006). In addition, two other studies reported that the severity of PTSD symptoms was negatively correlated with mPFC activity (Shin et al., 2005, Hopper et al., 2007). Moreover, a recent study investigated the relationship between default mode network connectivity and the severity of PTSD symptoms in subjects who had experienced an acute traumatic event 6-12 weeks before. The results of this study suggest that the resting-state connectivity of the posterior cingulate cortex/precuneus with the perigenual ACC and right amygdala is associated with current PTSD symptoms and that the correlation with the right amygdala predicts future PTSD symptoms (Lanius et al., 2010). Another study measuring resting-state amygdala connectivity in male veterans with PTSD $(n=15)$ and in combat controls $(n=14)$ showed greater positive connectivity between the amygdala and the insula 
in patients with PTSD than in controls, reduced positive connectivity between the amygdala and the hippocampus, and a reduced anticorrelation between the amygdala and the dorsal and rostral ACC (Sripada et al., 2012).

However, the mechanism underlying the alteration in connectivity between amygdala and prefrontal cortex in trauma-related psychiatric disorders remains unclear. Several lines of evidence have indicated that cortisol could be implicated (Henckens et al., 2010, Kern et al., 2008, Urry et al., 2006, Veer et al., 2012). The strength of the resting-state functional connectivity between the amygdala and the MPFC may be related to individual differences in endogenous cortisol levels under relatively stress-free circumstances (Veer et al., 2012). There is an adaptive mechanism of time-dependent modulation of the amygdala by corticosteroids: rapid nongenomic effects of corticosteroids suppress overall amygdala activity in a nonspecific manner, whereas slow genomic actions of corticosteroids normalize responses to negative input by specifically altering prefrontal control (Henckens et al., 2010). It has been suggested that serotonin (5-hydroxytryptamine) also plays a role in regulating functional interactions between the amygdala and the prefrontal cortex (Davidson et al., 2000, Siever, 2008). A recent neuroimaging study using two complementary methods (psycho-physiological-interaction in a general linear model and dynamic causal modeling) demonstrated that acute tryptophan depletion significantly altered the functional connectivity between the amygdala and the right ventral ACC and the vIPFC when processing angry vs. neutral faces (Passamonti et al., 2012).

\subsection{Recovery and the amygdala-prefrontal connectivity}

Interestingly, recent findings raise the possibility that more efficient crosstalk between the amygdala and the prefrontal cortex predicts beneficial behavioral outcomes in terms of emotion regulation and anxiety (Kim et al., 2011). It has been found that activity in specific areas of the frontal cortex (dorsolateral, dorsal medial, anterior cingulate, orbital) varies together with amygdala activity and that this functional connectivity is dependent on the reappraisal task. Moreover, the strength of coupling between the amygdala and the OFC/dorsal medial prefrontal cortex predicts the extent of attenuation of negative affect following reappraisal (Banks et al., 2007). In addition, a recent fMRI study investigating the effect of psychotherapy on coping demonstrated that a marked increased in mPFC activity accompanied decreased amygdala activity during traumatic memory retrieval in partial PTSD policemen $(n=12)$ after psychotherapy and these findings were associated with symptom attenuation (Peres et al., 2011).

\section{Conclusion}

Advances in neuroimaging technology have opened up new ways of understanding human brain function. Numerous studies indicate that the amygdala and the prefrontal cortex are important neuroanatomical structures involved in the response to traumatic stress and its effects on learning and memory. In particular, the amygdala and the prefrontal cortex are structurally and functionally interconnected. Traumatic experiences can be associated with 
lasting changes in these brain areas. These changes may result in an imbalance between the amygdala and the prefrontal cortex that is typically characterized by hyperactivity of the amygdala and hypoactivity of the prefrontal cortex. Investigations of the connection between the amygdala and prefrontal cortex have provided a deeper understanding of the role of the amygdala-prefrontal circuitry in trauma-related psychiatric disorders. Restoring the imbalance between hyperactivity of the amygdala and hypoactivity of the prefrontal cortex - in the form of robust structural and functional connectivity between the amygdala and the prefrontal cortex - predicts beneficial outcomes in the treatment of trauma-related psychiatric disorders.

\section{Author details}

\section{Dong Hoon Oh}

Department of Psychiatry, College of Medicine and Institute of Mental Health, Hanyang University, Seoul, Republic of Korea

\section{References}

[1] Akirav, I. \& Maroun, M. 2007. The role of the medial prefrontal cortex-amygdala circuit in stress effects on the extinction of fear. Neural Plast, 2007, 30873.

[2] American Psychiatric Association. \& American Psychiatric Association. Task Force On DSM-IV. 1994. Diagnostic and statistical manual of mental disorders : DSM-IV, Washington, DC, American Psychiatric Association.

[3] Ansell, E. B., Rando, K., Tuit, K., Guarnaccia, J. \& Sinha, R. 2012. Cumulative Adversity and Smaller Gray Matter Volume in Medial Prefrontal, Anterior Cingulate, and Insula Regions. Biol Psychiatry, 72, 57-64.

[4] Banks, S. J., Eddy, K. T., Angstadt, M., Nathan, P. J. \& Phan, K. L. 2007. Amygdalafrontal connectivity during emotion regulation. Soc Cogn Affect Neurosci, 2, 303-12.

[5] Bishop, S. J. 2007. Neurocognitive mechanisms of anxiety: an integrative account. Trends Cogn Sci, 11, 307-16.

[6] Bora, E., Harrison, B. J., Davey, C. G., Yucel, M. \& Pantelis, C. 2012. Meta-analysis of volumetric abnormalities in cortico-striatal-pallidal-thalamic circuits in major depressive disorder. Psychol Med, 42, 671-81.

[7] Bremner, J. D. 2006. Traumatic stress: effects on the brain. Dialogues Clin Neurosci, 8, 44561.

[8] Breslau, N. 2002. Epidemiologic studies of trauma, posttraumatic stress disorder, and other psychiatric disorders. Can J Psychiatry, 47, 923-9.

[9] Breslau, N., Kessler, R. C., Chilcoat, H. D., Schultz, L. R., Davis, G. C. \& Andreski, P. 1998. Trauma and posttraumatic stress disorder in the community: the 1996 Detroit Area Survey of Trauma. Arch Gen Psychiatry, 55, 626-32.

[10] Carrion, V. G., Garrett, A., Menon, V., Weems, C. F. \& Reiss, A. L. 2008. Posttraumatic stress symptoms and brain function during a response-inhibition task: an fMRI study in youth. Depress Anxiety, 25, 514-26. 
[11] Copeland, W. E., Keeler, G., Angold, A. \& Costello, E. J. 2007. Traumatic events and posttraumatic stress in childhood. Arch Gen Psychiatry, 64, 577-84.

[12] Croxson, P. L., Johansen-Berg, H., Behrens, T. E., Robson, M. D., Pinsk, M. A., Gross, C. G., Richter, W., Richter, M. C., Kastner, S. \& Rushworth, M. F. 2005. Quantitative investigation of connections of the prefrontal cortex in the human and macaque using probabilistic diffusion tractography. J Neurosci, 25, 8854-66.

[13] Dannlowski, U., Ohrmann, P., Konrad, C., Domschke, K., Bauer, J., Kugel, H., Hohoff, C., Schoning, S., Kersting, A., Baune, B. T., Mortensen, L. S., Arolt, V., Zwitserlood, P., Deckert, J., Heindel, W. \& Suslow, T. 2009. Reduced amygdala-prefrontal coupling in major depression: association with MAOA genotype and illness severity. Int $J$ Neuropsychopharmacol, 12, 11-22.

[14] Dannlowski, U., Stuhrmann, A., Beutelmann, V., Zwanzger, P., Lenzen, T., Grotegerd, D., Domschke, K., Hohoff, C., Ohrmann, P., Bauer, J., Lindner, C., Postert, C., Konrad, C., Arolt, V., Heindel, W., Suslow, T. \& Kugel, H. 2012. Limbic scars: long-term consequences of childhood maltreatment revealed by functional and structural magnetic resonance imaging. Biol Psychiatry, 71, 286-93.

[15] Davidson, R. J., Putnam, K. M. \& Larson, C. L. 2000. Dysfunction in the neural circuitry of emotion regulation--a possible prelude to violence. Science, 289, 591-4.

[16] De Vries, G. J. \& Olff, M. 2009. The lifetime prevalence of traumatic events and posttraumatic stress disorder in the Netherlands. J Trauma Stress, 22, 259-67.

[17] Driessen, M., Beblo, T., Mertens, M., Piefke, M., Rullkoetter, N., Silva-Saavedra, A., Reddemann, L., Rau, H., Markowitsch, H. J., Wulff, H., Lange, W. \& Woermann, F. G. 2004. Posttraumatic stress disorder and fMRI activation patterns of traumatic memory in patients with borderline personality disorder. Biol Psychiatry, 55, 603-11.

[18] Etkin, A., Klemenhagen, K. C., Dudman, J. T., Rogan, M. T., Hen, R., Kandel, E. R. \& Hirsch, J. 2004. Individual differences in trait anxiety predict the response of the basolateral amygdala to unconsciously processed fearful faces. Neuron, 44, 1043-55.

[19] Etkin, A. \& Wager, T. D. 2007. Functional neuroimaging of anxiety: a meta-analysis of emotional processing in PTSD, social anxiety disorder, and specific phobia. Am J Psychiatry, 164, 1476-88.

[20] Finkelhor, D., Ormrod, R., Turner, H. \& Hamby, S. L. 2005. The victimization of children and youth: a comprehensive, national survey. Child Maltreat, 10, 5-25.

[21] Foland-Ross, L. C., Altshuler, L. L., Bookheimer, S. Y., Lieberman, M. D., Townsend, J., Penfold, C., Moody, T., Ahlf, K., Shen, J. K., Madsen, S. K., Rasser, P. E., Toga, A. W. \& Thompson, P. M. 2010. Amygdala reactivity in healthy adults is correlated with prefrontal cortical thickness. J Neurosci, 30, 16673-8.

[22] Francati, V., Vermetten, E. \& Bremner, J. D. 2007. Functional neuroimaging studies in posttraumatic stress disorder: review of current methods and findings. Depress Anxiety, 24, 202-18.

[23] Frans, O., Rimmo, P. A., Aberg, L. \& Fredrikson, M. 2005. Trauma exposure and posttraumatic stress disorder in the general population. Acta Psychiatr Scand, 111, 291-9. 
[24] Ganzel, B. L., Kim, P., Glover, G. H. \& Temple, E. 2008. Resilience after 9/11: multimodal neuroimaging evidence for stress-related change in the healthy adult brain. Neuroimage, 40, 788-95.

[25] Gilbert, R., Widom, C. S., Browne, K., Fergusson, D., Webb, E. \& Janson, S. 2009. Burden and consequences of child maltreatment in high-income countries. Lancet, 373, 68-81.

[26] Grant, M. M., Cannistraci, C., Hollon, S. D., Gore, J. \& Shelton, R. 2011. Childhood trauma history differentiates amygdala response to sad faces within MDD. J Psychiatr Res, 45, 886-95.

[27] Hakamata, Y., Matsuoka, Y., Inagaki, M., Nagamine, M., Hara, E., Imoto, S., Murakami, K., Kim, Y. \& Uchitomi, Y. 2007. Structure of orbitofrontal cortex and its longitudinal course in cancer-related post-traumatic stress disorder. Neurosci Res, 59, 383-9.

[28] Helzer, J. E., Robins, L. N. \& Mcevoy, L. 1987. Post-traumatic stress disorder in the general population. Findings of the epidemiologic catchment area survey. $N$ Engl J Med, 317, 1630-4.

[29] Henckens, M. J., Van Wingen, G. A., Joels, M. \& Fernandez, G. 2010. Time-dependent effects of corticosteroids on human amygdala processing. J Neurosci, 30, 12725-32.

[30] Hopper, J. W., Frewen, P. A., Van Der Kolk, B. A. \& Lanius, R. A. 2007. Neural correlates of reexperiencing, avoidance, and dissociation in PTSD: symptom dimensions and emotion dysregulation in responses to script-driven trauma imagery. J Trauma Stress, 20, 713-25.

[31] Hsu, D. T., Langenecker, S. A., Kennedy, S. E., Zubieta, J. K. \& Heitzeg, M. M. 2010. fMRI BOLD responses to negative stimuli in the prefrontal cortex are dependent on levels of recent negative life stress in major depressive disorder. Psychiatry Res, 183, 202-8.

[32] Ichihara-Takeda, S. \& Funahashi, S. 2007. Activity of primate orbitofrontal and dorsolateral prefrontal neurons: task-related activity during an oculomotor delayedresponse task. Exp Brain Res, 181, 409-25.

[33] Karl, A., Schaefer, M., Malta, L. S., Dorfel, D., Rohleder, N. \& Werner, A. 2006. A metaanalysis of structural brain abnormalities in PTSD. Neurosci Biobehav Rev, 30, 1004-31.

[34] Kern, S., Oakes, T. R., Stone, C. K., Mcauliff, E. M., Kirschbaum, C. \& Davidson, R. J. 2008. Glucose metabolic changes in the prefrontal cortex are associated with HPA axis response to a psychosocial stressor. Psychoneuroendocrinology, 33, 517-29.

[35] Kim, M. J., Gee, D. G., Loucks, R. A., Davis, F. C. \& Whalen, P. J. 2011. Anxiety dissociates dorsal and ventral medial prefrontal cortex functional connectivity with the amygdala at rest. Cereb Cortex, 21, 1667-73.

[36] Kim, M. J. \& Whalen, P. J. 2009. The structural integrity of an amygdala-prefrontal pathway predicts trait anxiety. J Neurosci, 29, 11614-8.

[37] Koenigs, M. \& Grafman, J. 2009. Posttraumatic stress disorder: the role of medial prefrontal cortex and amygdala. Neuroscientist, 15, 540-8.

[38] Kolassa, I. T., Wienbruch, C., Neuner, F., Schauer, M., Ruf, M., Odenwald, M. \& Elbert, T. 2007. Altered oscillatory brain dynamics after repeated traumatic stress. BMC Psychiatry, 7, 56. 
[39] Lanius, R. A., Bluhm, R. L., Coupland, N. J., Hegadoren, K. M., Rowe, B., Theberge, J., Neufeld, R. W., Williamson, P. C. \& Brimson, M. 2010. Default mode network connectivity as a predictor of post-traumatic stress disorder symptom severity in acutely traumatized subjects. Acta Psychiatr Scand, 121, 33-40.

[40] Lupien, S. J., Parent, S., Evans, A. C., Tremblay, R. E., Zelazo, P. D., Corbo, V., Pruessner, J. C. \& Seguin, J. R. 2011. Larger amygdala but no change in hippocampal volume in 10-year-old children exposed to maternal depressive symptomatology since birth. Proc Natl Acad Sci U S A, 108, 14324-9.

[41] Lyoo, I. K., Kim, J. E., Yoon, S. J., Hwang, J., Bae, S. \& Kim, D. J. 2011. The neurobiological role of the dorsolateral prefrontal cortex in recovery from trauma. Longitudinal brain imaging study among survivors of the South Korean subway disaster. Arch Gen Psychiatry, 68, 701-13.

[42] Matsuoka, Y., Yamawaki, S., Inagaki, M., Akechi, T. \& Uchitomi, Y. 2003. A volumetric study of amygdala in cancer survivors with intrusive recollections. Biol Psychiatry, 54, 736-43.

[43] Mccrory, E., De Brito, S. A. \& Viding, E. 2011. The impact of childhood maltreatment: a review of neurobiological and genetic factors. Front Psychiatry, 2, 48.

[44] Mcfarlane, A. C., Yehuda, R. \& Clark, C. R. 2002. Biologic models of traumatic memories and post-traumatic stress disorder. The role of neural networks. Psychiatr Clin North Am, 25, 253-70, v.

[45] Mehta, M. A., Golembo, N. I., Nosarti, C., Colvert, E., Mota, A., Williams, S. C., Rutter, M. \& Sonuga-Barke, E. J. 2009. Amygdala, hippocampal and corpus callosum size following severe early institutional deprivation: the English and Romanian Adoptees study pilot. J Child Psychol Psychiatry, 50, 943-51.

[46] Mills, K. L., Mcfarlane, A. C., Slade, T., Creamer, M., Silove, D., Teesson, M. \& Bryant, R. 2011. Assessing the prevalence of trauma exposure in epidemiological surveys. Aust N Z J Psychiatry, 45, 407-15.

[47] Mollica, R. F., Lyoo, I. K., Chernoff, M. C., Bui, H. X., Lavelle, J., Yoon, S. J., Kim, J. E. \& Renshaw, P. F. 2009. Brain structural abnormalities and mental health sequelae in South Vietnamese ex-political detainees who survived traumatic head injury and torture. Arch Gen Psychiatry, 66, 1221-32.

[48] Mueller, S. C., Maheu, F. S., Dozier, M., Peloso, E., Mandell, D., Leibenluft, E., Pine, D. S. \& Ernst, M. 2010. Early-life stress is associated with impairment in cognitive control in adolescence: an fMRI study. Neuropsychologia, 48, 3037-44.

[49] New, A. S., Hazlett, E. A., Buchsbaum, M. S., Goodman, M., Mitelman, S. A., Newmark, R., Trisdorfer, R., Haznedar, M. M., Koenigsberg, H. W., Flory, J. \& Siever, L. J. 2007. Amygdala-prefrontal disconnection in borderline personality disorder. Neuropsychopharmacology, 32, 1629-40.

[50] Nunes, P. M., Wenzel, A., Borges, K. T., Porto, C. R., Caminha, R. M. \& De Oliveira, I. R. 2009. Volumes of the hippocampus and amygdala in patients with borderline personality disorder: a meta-analysis. J Pers Disord, 23, 333-45. 
[51] Oh, D. H., Son, H., Hwang, S. \& Kim, S. H. 2012. Neuropathological abnormalities of astrocytes, GABAergic neurons, and pyramidal neurons in the dorsolateral prefrontal cortices of patients with major depressive disorder. Eur Neuropsychopharmacol, 22, 330-8.

[52] Passamonti, L., Crockett, M. J., Apergis-Schoute, A. M., Clark, L., Rowe, J. B., Calder, A. J. \& ROBBINS, T. W. 2012. Effects of acute tryptophan depletion on prefrontalamygdala connectivity while viewing facial signals of aggression. Biol Psychiatry, 71, 3643.

[53] Peres JF, Foerster B, Santana LG, Fereira MD, Nasello AG, Savoia M, Moreira-Almeida A, Lederman H. 2011. Police officers under attack: resilience implications of an fMRI study. J Psychiatr Res,. 45, 727-34.

[54] Pessoa, L. 2010. Emotion and cognition and the amygdala: from "what is it?" to "what's to be done?". Neuropsychologia, 48, 3416-29.

[55] Phelps, E. A. \& Ledoux, J. E. 2005. Contributions of the amygdala to emotion processing: from animal models to human behavior. Neuron, 48, 175-87.

[56] Rauch, S. L., Whalen, P. J., Shin, L. M., Mcinerney, S. C., Macklin, M. L., Lasko, N. B., Orr, S. P. \& Pitman, R. K. 2000. Exaggerated amygdala response to masked facial stimuli in posttraumatic stress disorder: a functional MRI study. Biol Psychiatry, 47, 76976.

[57] Sacher, J., Neumann, J., Funfstuck, T., Soliman, A., Villringer, A. \& Schroeter, M. L. 2012. Mapping the depressed brain: A meta-analysis of structural and functional alterations in major depressive disorder. J Affect Disord. 140, 142-8

[58] Sah, P., Faber, E. S., Lopez De Armentia, M. \& Power, J. 2003. The amygdaloid complex: anatomy and physiology. Physiol Rev, 83, 803-34.

[59] Sehlmeyer, C., Dannlowski, U., Schoning, S., Kugel, H., Pyka, M., Pfleiderer, B., Zwitserlood, P., Schiffbauer, H., Heindel, W., Arolt, V. \& Konrad, C. 2011. Neural correlates of trait anxiety in fear extinction. Psychol Med, 41, 789-98.

[60] Sheline, Y. I., Barch, D. M., Donnelly, J. M., Ollinger, J. M., Snyder, A. Z. \& Mintun, M. A. 2001. Increased amygdala response to masked emotional faces in depressed subjects resolves with antidepressant treatment: an fMRI study. Biol Psychiatry, 50, 651-8.

[61] Shin, L. M., Rauch, S. L. \& Pitman, R. K. 2006. Amygdala, medial prefrontal cortex, and hippocampal function in PTSD. Ann N Y Acad Sci, 1071, 67-79.

[62] Shin, L. M., Wright, C. I., Cannistraro, P. A., Wedig, M. M., Mcmullin, K., Martis, B., Macklin, M. L., Lasko, N. B., Cavanagh, S. R., Krangel, T. S., Orr, S. P., Pitman, R. K., Whalen, P. J. \& Rauch, S. L. 2005. A functional magnetic resonance imaging study of amygdala and medial prefrontal cortex responses to overtly presented fearful faces in posttraumatic stress disorder. Arch Gen Psychiatry, 62, 273-81.

[63] Siegle, G. J., Thompson, W., Carter, C. S., Steinhauer, S. R. \& Thase, M. E. 2007. Increased amygdala and decreased dorsolateral prefrontal BOLD responses in unipolar depression: related and independent features. Biol Psychiatry, 61, 198-209.

[64] Siever, L. J. 2008. Neurobiology of aggression and violence. Am J Psychiatry, 165, 429-42.

[65] Sripada, R. K., King, A. P., Garfinkel, S. N., Wang, X., Sripada, C. S., Welsh, R. C. \& Liberzon, I. 2012. Altered resting-state amygdala functional connectivity in men with posttraumatic stress disorder. J Psychiatry Neurosci, 37, 241-9. 
[66] Suslow, T., Konrad, C., Kugel, H., Rumstadt, D., Zwitserlood, P., Schoning, S., Ohrmann, P., Bauer, J., Pyka, M., Kersting, A., Arolt, V., Heindel, W. \& Dannlowski, U. 2010. Automatic mood-congruent amygdala responses to masked facial expressions in major depression. Biol Psychiatry, 67, 155-60.

[67] Tomoda, A., Suzuki, H., Rabi, K., Sheu, Y. S., Polcari, A. \& Teicher, M. H. 2009. Reduced prefrontal cortical gray matter volume in young adults exposed to harsh corporal punishment. Neuroimage, 47 Suppl 2, T66-71.

[68] Tottenham, N., Hare, T. A., Quinn, B. T., Mccarry, T. W., Nurse, M., Gilhooly, T., Millner, A., Galvan, A., Davidson, M. C., Eigsti, I. M., Thomas, K. M., Freed, P. J., Booma, E. S., Gunnar, M. R., Altemus, M., Aronson, J. \& Casey, B. J. 2010. Prolonged institutional rearing is associated with atypically large amygdala volume and difficulties in emotion regulation. Dev Sci, 13, 46-61.

[69] Tottenham, N., Hare, T. A., Millner, A., Gilhooly, T., Zevin, J. D. \& Casey, B. J. 2011. Elevated amygdala response to faces following early deprivation. Dev Sci, 14, 190-204.

[70] Urry, H. L., Van Reekum, C. M., Johnstone, T., Kalin, N. H., Thurow, M. E., Schaefer, H. S., Jackson, C. A., Frye, C. J., Greischar, L. L., Alexander, A. L. \& Davidson, R. J. 2006. Amygdala and ventromedial prefrontal cortex are inversely coupled during regulation of negative affect and predict the diurnal pattern of cortisol secretion among older adults. J Neurosci, 26, 4415-25.

[71] Van Harmelen, A. L., Van Tol, M. J., Van Der Wee, N. J., Veltman, D. J., Aleman, A., Spinhoven, P., Van Buchem, M. A., Zitman, F. G., Penninx, B. W. \& Elzinga, B. M. 2010. Reduced medial prefrontal cortex volume in adults reporting childhood emotional maltreatment. Biol Psychiatry, 68, 832-8.

[72] Veer, I. M., Oei, N. Y., Spinhoven, P., Van Buchem, M. A., Elzinga, B. M. \& Rombouts, S. A. 2012. Endogenous cortisol is associated with functional connectivity between the amygdala and medial prefrontal cortex. Psychoneuroendocrinology, 37, 1039-47.

[73] Williams, L. M., Kemp, A. H., Felmingham, K., Barton, M., Olivieri, G., Peduto, A., Gordon, E. \& Bryant, R. A. 2006. Trauma modulates amygdala and medial prefrontal responses to consciously attended fear. Neuroimage, 29, 347-57.

[74] Woon, F. L. \& Hedges, D. W. 2008. Hippocampal and amygdala volumes in children and adults with childhood maltreatment-related posttraumatic stress disorder: a metaanalysis. Hippocampus, 18, 729-36.

[75] Woon, F. L. \& Hedges, D. W. 2009. Amygdala volume in adults with posttraumatic stress disorder: a meta-analysis. J Neuropsychiatry Clin Neurosci, 21, 5-12.

[76] Zhong, M., Wang, X., Xiao, J., Yi, J., Zhu, X., Liao, J., Wang, W. \& Yao, S. 2011. Amygdala hyperactivation and prefrontal hypoactivation in subjects with cognitive vulnerability to depression. Biol Psychol, 88, 233-42.

[77] Zhu, M. Y., Wang, W. P. \& Bissette, G. 2006. Neuroprotective effects of agmatine against cell damage caused by glucocorticoids in cultured rat hippocampal neurons. Neuroscience, 141, 2019-27. 